\title{
In vitro study on the impact of different cusp inclinations on cracked teeth
}

\author{
Nina Xie ${ }^{1,2}$, Zongxiang Liu ${ }^{2}$, Cui $\mathrm{Wu}^{2}$, Penglai Wang ${ }^{2}$, Guangtai Song ${ }^{2}$, Zhi Chen ${ }^{\text {* }}$ \\ ${ }^{1}$ Hubei Province Key Laboratory of Oral Biomedicine (Wuhan University), Ministry of Education Wuhan, Hubei, PR China \\ ${ }^{2}$ Xuzhou Stomatological Hospital Affiliated to Xuzhou Medical University, Xuzhou Jiangsu, PR China
}

\begin{abstract}
Objective: To study the relation between cracked teeth with different cusp inclination and maximum resistance strength.

Materials and methods: Seventy maxillary premolars were randomly divided into four groups. Groups 1,2 and 3 were made into models of cracked teeth with different buccal and palate cusp, respectively, while group 4 was the control group (untreated). Compression testing for the four groups was carried out using an electronic universal testing machine. The maximum resistance strength when the teeth cracked was recorded.

Results: Differences of the average maximum resistance strength of each group were statistically significant $(\mathbf{P}<\mathbf{0 . 0 5})$. Resistance strength decreased as cusp inclination increased. However, when cusp inclination increased to a specific level, there was no obvious decline.

Conclusions: The maximum resistance strength of different cusp inclinations is different, which decreases as cusp inclination increases.
\end{abstract}

Keywords: Cracked tooth syndrome; Cusp inclination; Maximum resistance strength.

Accepted on April 27, 2017

\section{Introduction}

Cracked tooth, known as cracked tooth syndrome, is the incomplete fracture of premolars or molar dental crown (sometimes the pulp is also affected due to the fracture) [1]. Epidemic studies have shown that cracked teeth have become the $3^{\text {rd }}$ major cause of tooth loss, after dental caries and periodontal disease in developing countries [2]. The clinical symptoms of cracked teeth become more severe as the crack depth increases. When the crack is comparatively deep, the prognosis is often poor. When the crack is slight, symptoms are not obvious. Thus, the prevention of crack teeth is extremely important. Currently, studies from home and aboard are focused on the symptoms of patients and treatment methodology while basic studies on root causes and pathogenesis, which have serious impact, are overlooked. In the present study, the compression test was carried out using an in vitro model of tooth cracking. The effect of different cusp inclinations on fracture and the risk of high inclination cusps are evaluated.

\section{Materials and Methods}

\section{Teeth}

According to Geurtsen et al. the lower molars were the most susceptible to crack formation, followed by maxillary molars and mandibular premolars which are the least susceptible [2].
In retrospective studies, Tamse et al. reported that premolars were the most susceptible to fracture [3]. Since maxillary molars were highly prone to fracture and easily available, maxillary molars were selected as the subject for this study.

The surgical clinic of Xuzhou Stomatological Hospital Affiliated to Xuzhou Medical University provided 70 teeth from 18-25 year old patients, that were removed for orthodontic purposes and who had signed the informed consent.

The inclusion criteria included: maxillary molars with mature roots. The exclusion criteria included: teeth that were too large or too small, dental caries, severely worn, cracked or with obvious defects; patients who underwent filing treatment or who underwent root canal therapy; teeth with $\leq 2$ roots; and root bending $>30^{\circ}[4]$.

The cusp inclination of teeth in vitro was measured using RVG digital X-ray film. The average buccal cusp was $49 \pm 1.6^{\circ}$, while the average palatal cusp was $40 \pm 1.9^{\circ}$. The $95 \%$ confidence intervals of buccal cusp and palatal cusp were $\left(48.6^{\circ}, 49.3^{\circ}\right)$ and $\left(39.7^{\circ}, 40.3^{\circ}\right)$, respectively, according to statistical analysis. To decrease deviation, 40 teeth, which fell into the $95 \%$ confidence intervals, were selected among the 70 teeth collected. The soft tissue and calculus were removed from the surface of the teeth, and were immersed in normal saline at room temperature for future use. 


\section{Apparatus and materials}

Ruler $(0.1 \mathrm{~mm})$, protractor, compass, $0.9 \%$ normal saline, RVG imaging system, head magnifier, e-ruler software, WSQ50 diamond outer-circle cutter (Shenyang Maike Material Manufacturing Equipment Co., Ltd). HY-0580 microcomputer control electronic universal testing machine (Shanghai Hengyi Sophisticated Apparatus Co., Ltd), electronic digital caliper (Tianjin Medical Instruments Company, Tianjin, Chian), denture base resins (Shanghai Medical Equipment Shareholdings Co., Ltd), and Imprint II Garant light-body (3M ESPE).

\section{Treatment for teeth in vitro}

Teeth were grouped as followed: the first 3 groups consisted of cracked teeth, separated by degree of buccal cusp and palatal cusp inclination. Group 4 was the control group (Table 1).

Table 1. Grouping of teeth according to cusp inclination.

\begin{tabular}{lll}
\hline Group & $\mathrm{n}$ & Description \\
\hline 1 & 10 & Buccal cusp inclination: $59^{\circ} ;$ palatal cusp inclination: $50^{\circ}$ \\
\hline 2 & 10 & Buccal cusp inclination: $69^{\circ} ;$ palatal cusp inclination: $60^{\circ}$ \\
\hline 3 & 10 & Buccal cusp inclination: $64^{\circ} ;$ palatal cusp inclination: $60^{\circ}$ \\
\hline 4 & 10 & The control group (without grinding) \\
\hline
\end{tabular}

Note: buccal and palatal cusp inclination was in line with the $95 \%$ confidence intervals.

\section{Tooth fracture model}

Yunzhu et al. measured the cusp inclination of cracked teeth. The results demonstrated that the average cusp inclinations of cracked teeth near the mid-buccal and tip of the tongue was larger than in the control group. The difference in cusp inclination of teeth near the buccal between the cracked and control group was $9.02^{\circ}$, and for teeth near the tip of the tongue and the control group was $9.29^{\circ}$ [5]. Therefore, in this study, $10^{\circ}, 15^{\circ}$ and $20^{\circ}$ were added to the normal cusp inclinations of cracked teeth, while the cusp inclination of the control group remained unchanged. The planar graph was prepared based on the height of the buccal tooth tip (the distance between the tooth tip and the horizon of the central pits and fissures) and the width (the distance between the tooth tip and the horizon of the longitudinal axis of the tooth). To ensure that the cusp inclination qualified, the distance to be adjusted in the planar graph was calculated. A caliper with accuracy of $0.02 \mu \mathrm{m}$ was used to mark the distance to be adjusted. High speed diamond burs were used to grind and adjust the cusp inclination and prepare the cracks as previously described [6]: \#700 fissure burs were used to prepare precracks with a width of $2 / 3$ of the near, far and middle crown diameter to the height of the cementoenamel junction. In the groups of cracked teeth and the control group, silicon rubber was used to emulate periodontal membrane and self-curing resin was used to emulate alveolar bone, which was to be embedded in the box (Figure 1).

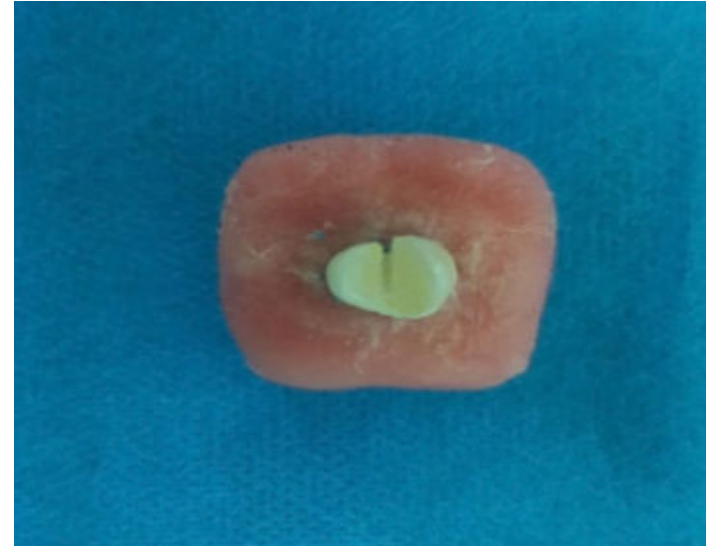

Figure 1. The embedded tooth.

\section{Defining the contact point for loading}

We carried out biting inspection on the plaster casting of cracked teeth preserved in the laboratory, using silicon rubber. The contact points of cracked teeth in the biting inspection were complex. The general rule was that the contact point in biting was usually in the $1 / 3$ mid-bottom part of the tooth slope. By measuring and analyzing the distance among tooth slopes, a $3.5 \mathrm{~mm}$-diameter stainless steel loading head was selected. The loading head would touch the buccal and palatal cusp and the contact point was around the 1/3 mid-bottom part of the tooth slope.

\section{Loading}

We carried out compression testing on all teeth using an electronic universal testing machine. The tooth was placed on the loading platform. The long axis was vertically erected on the horizontal plane so that the loading head vertically pressed on the defined contact point. Switch the apparatus and the loading head moved at a speed of $2 \mathrm{~mm} / \mathrm{min}$ until the tooth cracked. The computer automatically recorded the loading force at the time when the tooth cracked as the maximum resistance strength.

\section{Statistical analysis}

SPSS16.0 software was used for data analysis (to compare the maximum resistance strength among each group). Single factor t-test was used for comparisons between groups $(\alpha=0.05)$.

\section{Results}

\section{Maximum resistance strength}

Based on the comparison of the average resistance strength of each group by using the one-way analysis of variance, the differences in the average maximum resistance strength among the groups were statistically significant $(\mathrm{P}<0.05)$. The differences of the maximum resistance strength of group 1 and 4 and that of other groups were statistically significant, while 
the difference of group 2 and 3 was not statistically significant (Table 2).

Table 2. Rounds of comparison of the maximum resistance strength among all groups.

\begin{tabular}{lll}
\hline Group & $\mathrm{n}$ & $\overline{\mathrm{x}} \pm s(\mathrm{~N})$ \\
\hline 1 & 10 & $548.24 \pm 95.33^{\mathrm{a}}$ \\
\hline 2 & 10 & $387.52 \pm 58.46^{\mathrm{b}}$ \\
\hline 3 & 10 & $312.47 \pm 25.75^{\mathrm{b}}$ \\
\hline 4 & 10 & $1205.24 \pm 215.71^{\mathrm{c}}$
\end{tabular}

(Note: a-c: Characters were different, refers to statistically significant differences $(P<0.05)$.

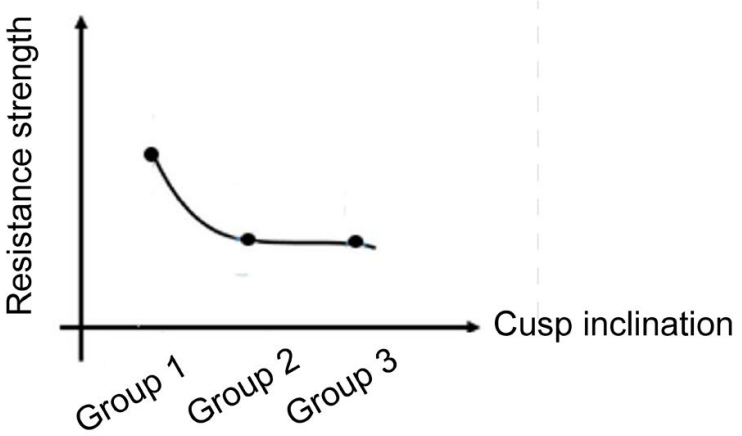

Figure 2. The relation between cusp inclination and resistance strength.

\section{The relation between cusp inclination and resistance strength}

The cusp inclinations of the experimental groups were at least $10^{\circ}$ larger than those of the control group while the resistance force of the experimental groups were smaller than those of the control group. The differences were statistically significant. Clinically, the cracking rate of cracked teeth was far greater than that of normal teeth, which was consistent with the results of this study [7].

The difference of buccal and palatal cusp inclination among the samples of the three experimental groups was $10^{\circ}$, respectively. The resistance strength of group 1 was larger than of group 2 and 3 , and the differences between groups 1, 2 and 3 were statistically significant $(\mathrm{P}<0.05)$. The difference of resistance strength between group 2 and 3 was not statistically significant $(\mathrm{P}>0.05)$. The resistance strength decreased as the cusp inclination increased. However, when the cusp inclination increased to a specific level, the resistance showed no obvious decrease with cusp inclination increase (Figure 2).

\section{Discussion}

In the present study, the RVG parallel projection method and a caliper were used to measure the anatomic shape of teeth, from which there were no differences with measurement by digital $\mathrm{X}$-ray film. Thus, the measurements we obtained are consistent with those by Hailiang et al. [8]. Compared with CT, RVG digital imaging is easier to operate, cheaper and more frequently used clinically. With parallel projection technology, the distortion rate is lower. It was therefore feasible for measuring cusp inclination in this study.

When chewing, biting force is diverted horizontally to the bottom of pits and fissures, due to the slope of teeth, which leads to cracks. Changes in cusp inclination lead to changes of the diverted horizontal force. Larger cusp inclination causes larger diverted horizontal force, which affects the possibility of cracking a tooth. Yunzhu et al. demonstrated that steeper cusp inclination and smaller tooth slope angle, increased the possibility of a tooth cracking [6]. When a tooth is cracked, its resistance strength is greatly decreased, which is consistent with the comparison of the resistance strength of cracked teeth in the experimental group and the control group.

However, the results also demonstrated there is an inverse relation between resistance strength and cusp inclination when the cusp inclination falls within a certain range. The primary reason is that the diverted horizontal force on the tooth increases as cusp inclination increases, which lead to higher possibility of a cracked tooth. However, when the cusp inclination increases to a certain level, the inverse relation between resistance strength and the cusp inclination disappears. A plateau phase will emerge, which might be a result of the outside-to-inside gradient distribution of teeth and the composition of teeth. Tooth composition is determined by the microstructure: the biting surface of the enamel rod is thick and short, that near the dentin-enamel junction is long and thin, and the enamel rods are interwoven together, enhancing the resistance of enamel against the biting force [9]. Additionally, the percentage of protein in the enamel decreases as its distance to the dentin interface increases. Protein can decrease the concentration of forces on the tip of the crack in the enamel and enhance the resistance capacity of the enamel against cracking [10]. This study demonstrates that when cusp inclination decreases to a certain angle, resistance strength is increased and the possibility of cracking will decrease.

\section{References}

1. Cameron CE. Cracked-tooth syndrome. J Am Dent Assoc 1964; 68: 405-411.

2. Geurtsen W, Schwarze T, Günay H. Diagnosis, therapy, and prevention of the cracked tooth syndrome[J]. Quintessence international (Berlin, Germany: 1985) 2003; 34: 409-417.

3. Tamse A, Zilburg I, Halpern J. Vertical root fractures in adjacent maxillary premolars: An endodontic-prosthetic perplexity. Int Endod J 1998; 31: 127-132.

4. Weirong T, Younong W, Ting W, Shenlin H. Study on the fracture resistance and fracture mode of different caviety in vitro root canal treatment. Stomatology 2011; 31: 219-223.

5. Yunzhu Q, Jia L, Libiao J. Measurement of threedimensional reconstruction cracked cusp inclination. J Clin Dent 2005; $21:$ 459-461. 
6. Liqun Z, Ye C, Yunpu S. Experimental study on the damage of the cracked teeth with $\mathrm{Ni}$ Ti shape memory alloy. Chin J of Conserv Dentist 2001; 11: 27-28.

7. Kang SH, Kim BS, Kim Y. Cracked teeth: Distribution, characteristics, and survival after root canal treatment. J Endod 2016; 42: 557-562.

8. Hailiang S, Yuxing B. Premolar periapical film bisecting angle protection and parallel comparison of tooth length. Beij J of Stomatol 2005; 13: 102-104.

9. Bajaj DAD. On the R-curve behavior of human tooth enamel. Biomaterials 2010; 30: 4037-4046.

10. Ji BH. A study of fracture mechanisms in biological nanocomposites via the virtual internal bondmodel. Mater Res A 2007; 81: 484-492.

\section{*Correspondence to}

Zhi Chen

Hubei Province Key Laboratory of Oral Biomedicine (Wuhan University),

Ministry of Education Wuhan,

Hubei, 4300079,

PR China 